\title{
Observation of a multiply ionized plasma with index of refraction greater than one
}

J. Filevich, J. J. Rocca, M. C. Marconi, S. J. Moon, J.

Nilsen, J. H. Scofield, J. Dunn, R. F. Smith, R. Keenan, J. R. Hunter, V. N. Shlyaptsev

October 15, 2004

Physical Review Letters 
This document was prepared as an account of work sponsored by an agency of the United States Government. Neither the United States Government nor the University of California nor any of their employees, makes any warranty, express or implied, or assumes any legal liability or responsibility for the accuracy, completeness, or usefulness of any information, apparatus, product, or process disclosed, or represents that its use would not infringe privately owned rights. Reference herein to any specific commercial product, process, or service by trade name, trademark, manufacturer, or otherwise, does not necessarily constitute or imply its endorsement, recommendation, or favoring by the United States Government or the University of California. The views and opinions of authors expressed herein do not necessarily state or reflect those of the United States Government or the University of California, and shall not be used for advertising or product endorsement purposes. 


\title{
Observation of a multiply ionized plasma with index of refraction greater than one
}

\author{
J. Filevich, J.J. Rocca, and M.C. Marconi \\ NSF ERC for Extreme Ultraviolet Science and Technology \\ and Department of Electrical and Computer Engineering, \\ Colorado State University, Fort Collins, Colorado 80523
}

S.J. Moon, J. Nilsen, J.H. Scofield, J. Dunn, R.F. Smith, R. Keenan, and J.R. Hunter Lawrence Livermore National Laboratory, Livermore, California 94550

\author{
V.N. Shlyaptsev \\ Department of Applied Science, University of California \\ Berkeley-Livermore, Livermore, California 94451
}

(Dated: October 12, 2004)

\begin{abstract}
We present clear experimental evidence showing that the contribution of bound electrons can dominate the index of refraction of laser created plasmas at soft x-ray wavelengths. We report anomalous fringe shifts in soft x-ray laser interferograms of Al laser-created plasmas. The comparison of measured and simulated interferograms show that this results from the dominant contribution of low charge ions to the index of refraction. This usually neglected bound electron contribution can affect the propagation of soft x-ray radiation in plasmas and the interferometric diagnostics of plasmas for many elements.
\end{abstract}

PACS numbers: 07.60.La, 42.87.Bg, 52.50.Jm, 52.25.Os, 52.25.Mq, 52.70.La

Keywords: 
The index of refraction is a very important characteristic of a plasma. It affects the propagation of self generated plasma radiation as well as that of the probe beams that are often used in plasma characterization techniques that include interferometry and refractometry. In partialy ionized plasmas, two color interferometry is often used to separate the contribution to the index of refraction of neutral atoms and free electrons [1]. In multiply ionized laser-created plasmas the index of refraction is usually calculated assuming that the contribution of bound electrons is negligible compared with that of free electrons. For example in all plasma interferometry experiments to date that used soft-x-ray laser probes, the spatial distribution of the electron density has been obtained assuming that the index of refraction is determined by the density of free electrons[2-8].

In this paper we present soft x-ray laser interferometry data obtained with a picosecond $14.7 \mathrm{~nm}(84.4 \mathrm{eV})$ laser probe that shows clear evidence of the dominant contribution of bound electrons to the index of refraction in the late stages of the evolution of an $\mathrm{Al}$ plasma created by a high power laser. The interferograms show that the fringes, late in the plasma evolution, in the periphery of the plasma and close to the target surface bend toward the target. In our experiment this direction of the fringe shifts is indicative of an index of refraction greater than 1 (hereafter referred as negative fringe shifts). In contrast, at earlier times all the fringes are observed to bend away from the target (positive fringe shifts). Similar negative fringe shifts were also recently observed in an independently realized $\mathrm{Al}$ laser-created plasma soft x-ray laser interferometry experiment at $13.9 \mathrm{~nm}$, and possible mechanisms were proposed but not analyzed in detail[9]. Analysis of our data, with the assistance of hydrodynamic model simulations, indicates that late in the plasma evolution the contribution of bound electrons dominates the index of refraction, causing the observed negative fringe shifts in the periphery regions of the plasma. The significance of the result goes beyond the particular case of aluminum, as this effect can strongly affect the index of refraction of many ionized materials at soft x-ray laser wavelengths and needs to be carefully considered when analyzing experiments.

The experiment was performed using a transient $14.7 \mathrm{~nm}$ Ni-like Pd soft x-ray laser [10] combined with an amplitude division diffraction grating interferometer (DGI) [5, 8]. The interferometer is set in a skewed Mach-Zehnder configuration, with the principal distinguishing characteristic being the use of diffraction gratings as beam splitters. The DGI design has proven to be robust and capable of producing interferograms with very good visibility over 
a large field of view, as first demonstrated with a $46.9 \mathrm{~nm}$ capillary discharge laser [5]. The Ni-like Pd soft x-ray laser used in this experiment produced laser pulses of few 10's of $\mu \mathrm{J}$ with a typical duration in the range of $4.5-5.2 \mathrm{ps}$ [11]. It was pumped by a sequence of a 600 ps FWHM, 1 J long pulse and a traveling wave 13 ps FWHM, 5 J short pulse generated by the Compact Multipulse Terawatt (COMET) chirped pulse amplification laser [10]. The resulting short duration of the soft x-ray laser pulse permits the acquisition of "snap-shots" of rapidly evolving plasmas, greatly reducing the blurring of interference fringes that occurs when the electron density profile changes significantly during the sampling period of the probe pulse.

Figure 1 shows interferograms of an expanding Al laser-produced plasma. The plasmas were heated at an irradiance of $10^{13} \mathrm{Wcm}^{-2}$ by focusing a $600 \mathrm{ps}, 3 \mathrm{~J}, 1054 \mathrm{~nm}$ laser into a $3.1 \mathrm{~mm}$ long $\times 12 \mu \mathrm{m}$ wide line focus on a $1 \mathrm{~mm}$ long flat Al target. The Al plasma was generated within the path of one arm of the interferometer, and the timing between the heating and probe beams was measured using a fast photodiode. The field of view of the interferograms, which is significantly larger than shown $(600 \mu \mathrm{m}$ by $400 \mu \mathrm{m})$, allows reliable reference fringes to be obtained. The two frames corresponding to the earlier time of the plasma evolution (0 and $0.8 \mathrm{~ns}$ ) show a rapid lateral expansion of the plasma together with the formation of an on-axis density depression. At these times the fringes shift away from the target, even in the central region of the plasma where the on-axis depression is observed. A similar two-dimensional feature was observed in soft x-ray laser interferograms of laser created $\mathrm{Cu}$ plasmas obtained using a $46.9 \mathrm{~nm}$ laser $[12,13]$. In that case the simulations showed that the electron density minimum in the central region of the plasma is the result of pressure equilibrium between the irradiated plasma region and the low temperature sidelobes, created by plasma-radiation induced ablation of the surrounding target region. The last two frames in Fig. 1 show an interferogram in which the region close to the target presents increased absorption, an indication of a colder plasma. Also the fringes at the periphery of the plasma shift toward the target, a phenomenon that requires a total index of refraction greater than 1 .

In phase shift interferometry the number of fringe shifts, given by $N_{f}=\frac{1}{\lambda} \int_{0}^{L}(1-\eta) d l$, is negative when the index of refraction $(\eta)$ of the plasma is greater than 1 . The contribution of the free electrons to the plasma index of refraction is always less than 1 , as determined by $\eta=\left(1-n_{e} / n_{\text {crit }}\right)^{\frac{1}{2}}$ where $n_{\text {crit }}=5 \times 10^{24} \mathrm{~cm}^{-3}$ is the critical density for $\lambda=14.7 \mathrm{~nm}$. 
This suggests that the contribution of bound electrons to $\eta$ is dominant and greater than one, in the region where negative fringe shifts are observed. The effect was not observed in interferograms of plasmas we generated with similar plasma heating beam conditions using several other target materials ( $\mathrm{Ti}, \mathrm{Cr}, \mathrm{Pd}, \mathrm{Mo}, \mathrm{Au}$ ). As an example, Fig. 2 shows interferograms for $\mathrm{Ti}$ and $\mathrm{Pd}$ plasmas, obtained at approximately the same late time in the plasma evolution as the last frame in Fig. 1, 3 ns after the peak of the heating laser pulse. While the corresponding Al interferogram shows negative fringe shifts on the periphery of the plasma, the Ti and Pd interferograms show only positive fringe shifts. A possible explanation for this difference can be found reviewing the values of the neutral atom's real component of the scattering factor $f_{1}^{0}$ at $14.7 \mathrm{~nm}$. From all elements with atomic number (Z) less than 54 , $\mathrm{Al}$ is the only one that has a negative $f_{1}^{0}[14,15]$ due to the close proximity of the $84.4 \mathrm{eV}$ photon energy to the Al L-shell absorption edge. Some elements with Z $\geqslant 54$, including I, Xe, $\mathrm{Cs}, \mathrm{Ba}, \mathrm{Fr}, \mathrm{Ra}, \mathrm{Ac}$, also have a negative scattering factor at this wavelength. This negative scattering factor translates into an index of refraction greater than one $\left(\eta=\left(1-\frac{r_{e} \lambda^{2} n_{a} f_{1}^{0}}{\pi}\right)^{\frac{1}{2}}\right.$ where $n_{a}$ is the density of neutral atoms, $\lambda$ is the probe beam wavelength and $r_{e}$ is the classical electron radius). This suggests that significant densities of neutral Al atoms, for which the L-shell electrons have a significant contribution to $\eta$, will cause negative fringe shifts. However, the high photoionization cross section of neutral Al and the fact that the probe beam is not completely absorbed in the region where negative fringe shifts are observed indicate that the neutral density of this plasma should be negligible and that the negative fringe shifts are in fact caused by bound electrons on ions. Therefore, the real component of the index of refraction $f_{1 Z}^{0}$ was computed for the different ions present in the plasma. For each ionization stage the continuum absorption cross sections were calculated using a Hartree-Slater code. The potentials were adjusted to make sure the L3 edges agree with the experimentally measured edges[16, 17]. The absorption for the lines below the L3 edges were subsequently added to the continuum absorption. For Al II and Al III the measured line positions and oscillator strengths from Aguilar et al. [16] were used. For Al IV the n=2 to $\mathrm{n}=3$ and $2 \mathrm{p}$ to $4 \mathrm{~s}$ line positions and line strengths were from Savukov et al. [17] Using the absorption coefficient, $f_{2}^{0}$, the real part of the index, $f_{1}^{0}$, is then derived using the KramersKronig dispersion relation. This part of the index is the bound electron contribution, so then the number of free electron was added to determine the total $f_{1}^{0}$, as discussed by Henke et al. [14]. It should be noted that the resonance line contributions are extremely sensitive to 
the line positions and should include the effects of line broadening. The calculation uses the best data available at this time with the possibility of further improvements in the future. We define the "effective" $f_{Z}^{*}$ value as $f_{Z}^{\text {bound }}+f_{Z}^{\text {free }}$ that includes the separate contributions of free and bound electrons, where $f_{Z}^{\text {free }}$ is the number of free electrons corresponding to the ion of charge $\mathrm{Z}$ with the forward scattering supraindex (0) and real part (1) subindex omitted. We calculated the values for $\mathrm{Al} \mathrm{I}-\mathrm{Al}$ IX to be [18]: $\mathrm{Al}$ I $=-0.85, \mathrm{Al}$ II $=-4.19$, $\mathrm{Al} \mathrm{III}=-3.54, \mathrm{Al} \mathrm{IV}=-1.80, \mathrm{Al} \mathrm{V}=0.84, \mathrm{Al} \mathrm{VI}=3.54, \mathrm{Al} \mathrm{VII}=5.30, \mathrm{Al} \mathrm{VIII}=6.73, \mathrm{Al} \mathrm{IX}$ $=8$. When the $f_{Z}^{*}$ values differ from $\mathrm{Z}$ (the number of free electrons corresponding to that ion) the contribution of the bound electrons is significant. These $f_{Z}^{*}$ values were used to calculate the total plasma index of refraction $\eta=\left(1-\sum_{Z} \frac{n_{Z} f_{Z}^{*}}{n_{\text {crit }}}\right)^{\frac{1}{2}}$, where $n_{Z}$ is the density of ions with charge Z obtained from the LASNEX simulations. All ions with charge up to $\mathrm{Z}=5$ were found to make a significant negative contribution to the index of refraction. The computed negative values of $f_{Z}^{*}$ for Al I-Al IV suggest that the negative fringe shifts observed at the late times are due to the presence of a relatively high densities of these low charge atoms. Moreover, notice that ions up to Al VII make a significant contribution to the index of refraction. It should be noticed that this phenomena does not require a close resonance and affects a broad range of wavelengths due to numerous bound-bound and bound free transitions in many ionization stages.

To confirm this interpretation of the data, the LASNEX hydrocode [19] was used to compute the 2-dimensional (2-D) ion and electron density distributions for the plasmas of Fig. 1. Simulations were performed using flux-limited heat transport, multi-group radiation diffusion, and a detailed hydrogenic configuration atomic model with non-LTE physics for temperatures above $50 \mathrm{eV}$. The simulations were conducted using the measured temporal and spatial profiles of the laser beam used to heat the plasma. Figure 3 shows 2-D maps of the computed electron density (contours) and the corresponding average ion charge distributions. The electron density distributions measured during the early times of the expansion, including the lateral expansion and on-axis depression, are well reproduced by the code, and will be discussed in a future publication. This indicates that the assumption that the free electrons dominate the contribution to the index of refraction is valid at the early times, as is expected from the fact that at those times the plasma is hot and only highly ionized species are present. At later times, corresponding to the last two frames of Fig. 1, the simulations show that the degree of ionization in the plasma periphery decreases 
as the plasma cools, resulting in significant concentrations of $\mathrm{Al}$ II-Al IV ions present. The simulation results were used to synthesize the interferograms that are shown in Fig 4 . These synthetic interferograms were calculated taking into account the contributions to the index of refraction from free electrons and $\mathrm{Al}$ ions using the computed "effective" $f_{Z}^{*}$ factors and the electron density and ion density distributions computed with LASNEX. The synthesized interferogram corresponding to 0.8 ns delay shows the central density depression and the lateral expansion observed in the measurements, and the late interferogram at 3.2 ns shows the observed negative fringe shifts in the periphery of the plasma, in good agreement with the experiment. In the simulations, the negative fringe shifts appear slightly earlier (at 0.8 $\mathrm{ns})$ than in the experiment (1.1 ns).

It should be expected that interferograms of the late stages of the evolution of many other laser-created plasmas involving other materials will also be significantly affected by the contribution of bound electrons. Moreover, the importance of the bound electron contribution is not always limited to plasmas with a low mean ion charge. For example, ten times ionized $\mathrm{Pd}$ atoms are computed to have an $f_{1}^{*}$ value that is several times that of the corresponding number of free electrons, with the precise value of $f_{1}^{*}$ depending strongly on the position of the resonance line[18]. It is also important to realize that the relative contribution of bound electron scattering is not always clearly evident in soft x-ray interferograms because at these wavelengths the free and bound electrons often contribute with the same sign to the phase delay. While soft x-ray laser interferometry allows measurements of higher plasma densities because of better spatial resolution, reduced absorption and reduced deflection angles within steep plasma density gradients, neglecting the contribution of bound electrons to the index of refraction could, in some cases, constitute a significant systematic error in the determination of the electron density.

In conclusion, we have demonstrated that bound electron can contribute significantly to the index of refraction of multiply ionized plasmas at soft x-ray wavelengths in the vicinity of absorption edges. In the case of an $\mathrm{Al}$ plasma the effect causes a dramatic change of sign of the fringe shifts in interferograms aquired using $\lambda=14.7 \mathrm{~nm}$ radiation. The significance of the result goes beyond the particular case of aluminum, as it can significantly affect the index of refraction of many materials at soft x-ray laser wavelengths. 


\section{Acknowledgments}

The authors thank Albert Osterheld for continued support. This work was sponsored by the National Nuclear Security Administration under the Stewardship Science Academic Alliances program through DOE Research Grant \# DE-FG03-02NA00062. Part of this

work was performed under the auspices of the U.S. Dept. of Energy by the University of California, Lawrence Livermore National Laboratory through the Institute of Laser Science and Application, under contract No. W-7405-Eng-48. The CSU researchers also gratefully acknowledge the partial support of the NSF ERC Center for Extreme Ultraviolet Science and Technology, award EEC-0310717.

[1] R. A. Alpher D. R. White Plasma diagnostic techniques Chapter 10, pages 441443,Academic Press (1965).

[2] L. B. Da Silva et al., Physical Review Letters 74, 3991 (1995).

[3] J. J. Rocca, C. H. Moreno, M. C. Marconi, and K. Kanizay, Optics Letters 24, 420 (1999).

[4] C. H. Moreno et al., Physical Review E 60, 911 (1999).

[5] J. Filevich et al., Optics Letters 25, 356 (2000).

[6] R. F. Smith et al., Physical Review Letters 89, 065004 (2002).

[7] B. Rus, et al., in $8^{\text {th }}$ International Conference on X-Ray Lasers, edited by J. Rocca (2002), pp. $182-189$.

[8] J. Filevich et al., Applied Optics 43 (2004).

[9] H. Tang et al., Applied Physics B 78, 975 (2004).

[10] J. Dunn et al., Physical Review Letters 84, 4834 (2000).

[11] J. Dunn et al., in SPIE Int. Soc. Opt. Eng. Proc, edited by E. E. Fill and S. Suckewer (2003), vol. 5197, pp. 51-59.

[12] J. Filevich et al., Physical Review E 67, 056409 (2003).

[13] J. J. Rocca et al., Physics of Plasmas 10, 2031 (2003).

[14] B. L. Henke, E. M. Gullikson, and J. C. Davis, Atomic Data and Nuclear Data Tables 54, $181(1993)$.

[15] E. M. Gullikson, P. Denham, S. Mrowka, and J. H. Underwood, Physical Review B 49, 16283 

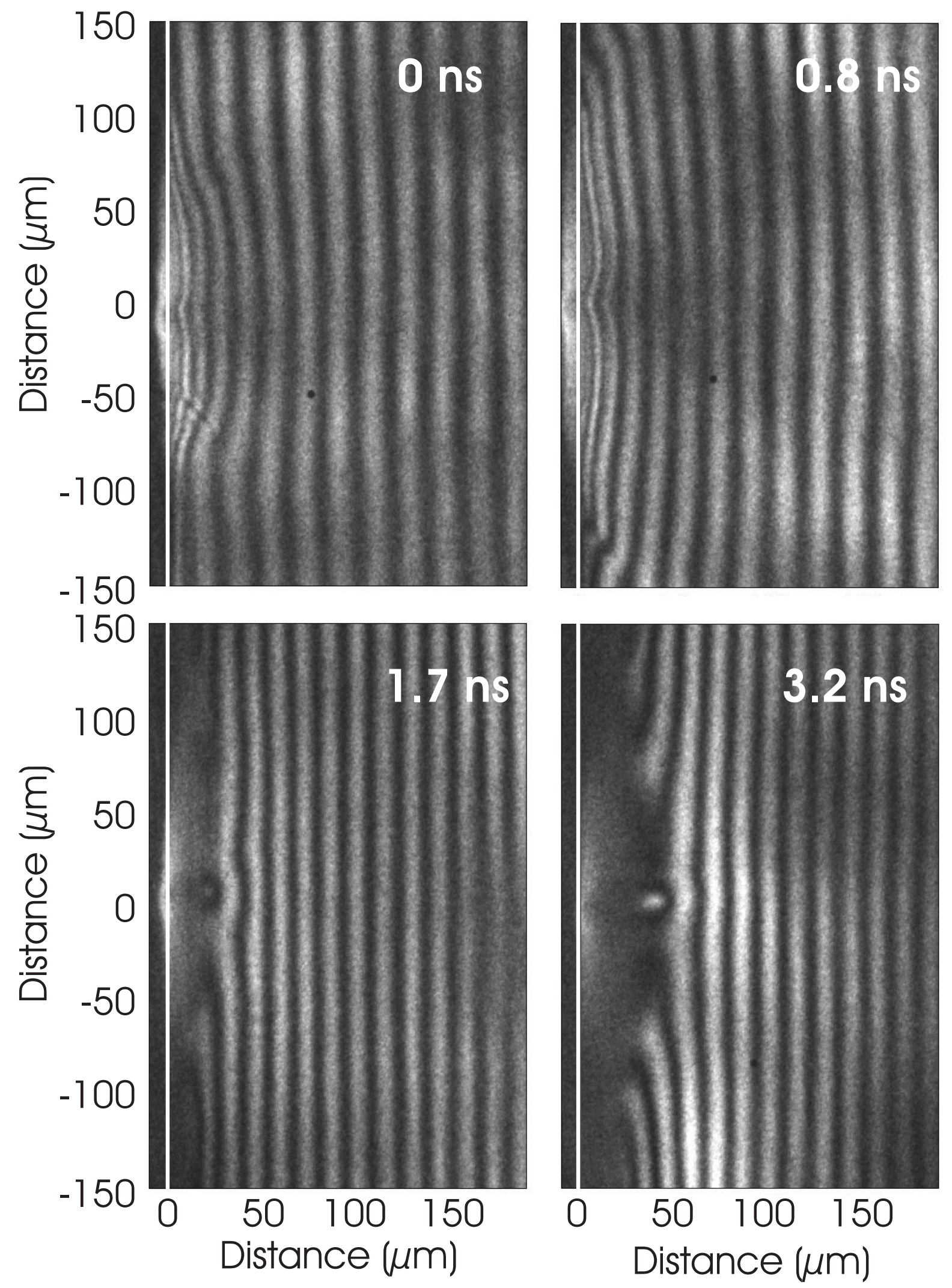

FIG. 1: Sequence of soft x-ray laser interferograms $(\lambda=14.7 \mathrm{~nm})$ of Al line focus plasmas. The plasmas were generated by a $3 \mathrm{~J}$ heating beam focused into a $12 \mu \mathrm{m} \times 1 \mathrm{~mm}$ line focus. The times are measured respect to the peak of the 600 ps heating pulse. Fringes that bend toward the target (negative fringe shifts)are observed in the last two frames in the periphery of the plasma and close to the target. 

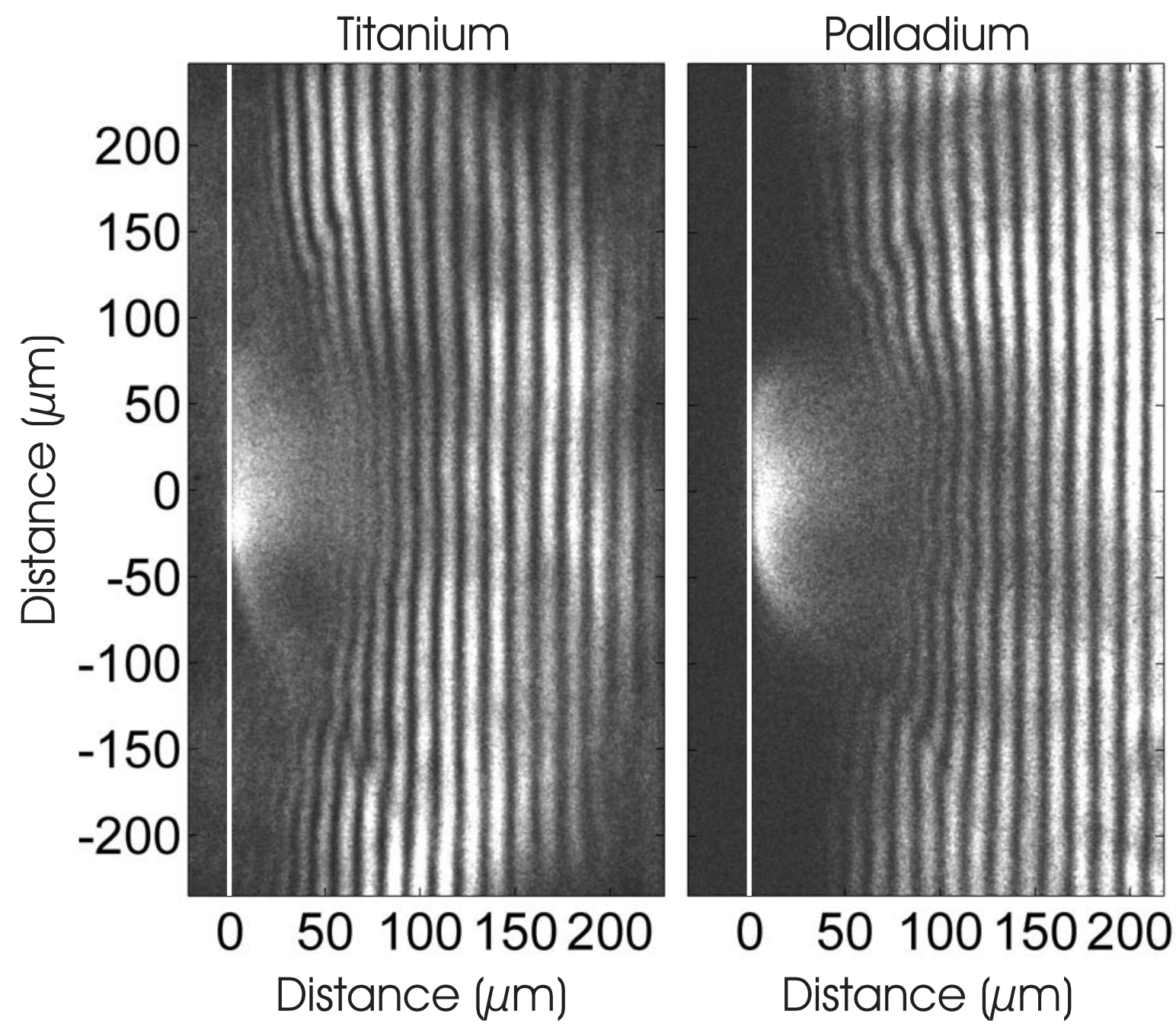

FIG. 2: Comparison of interferograms obtained 3 ns after the peak of the heating laser pulse for $\mathrm{Ti}$ and $\mathrm{Pd}$ targtes for heating conditions similar as those used to obtain the Al interferograms in Fig. 1. (1994).

[16] A. Aguilaret al., Physical Review A 67, 012701 (2003).

[17] I. M. Savukov, Journal of Physics B: Atomic, Molecular and Optical Physics 36, 4789 (2003).

[18] J. Nilsen and J. H. Scofield, UCRL-PROC-204652 (2004), livermore, CA.

[19] G. D. Zimmerman and W. L. Kruer, Comments Plasma Phys. Controlled Fusion 2, 51 (1975). 


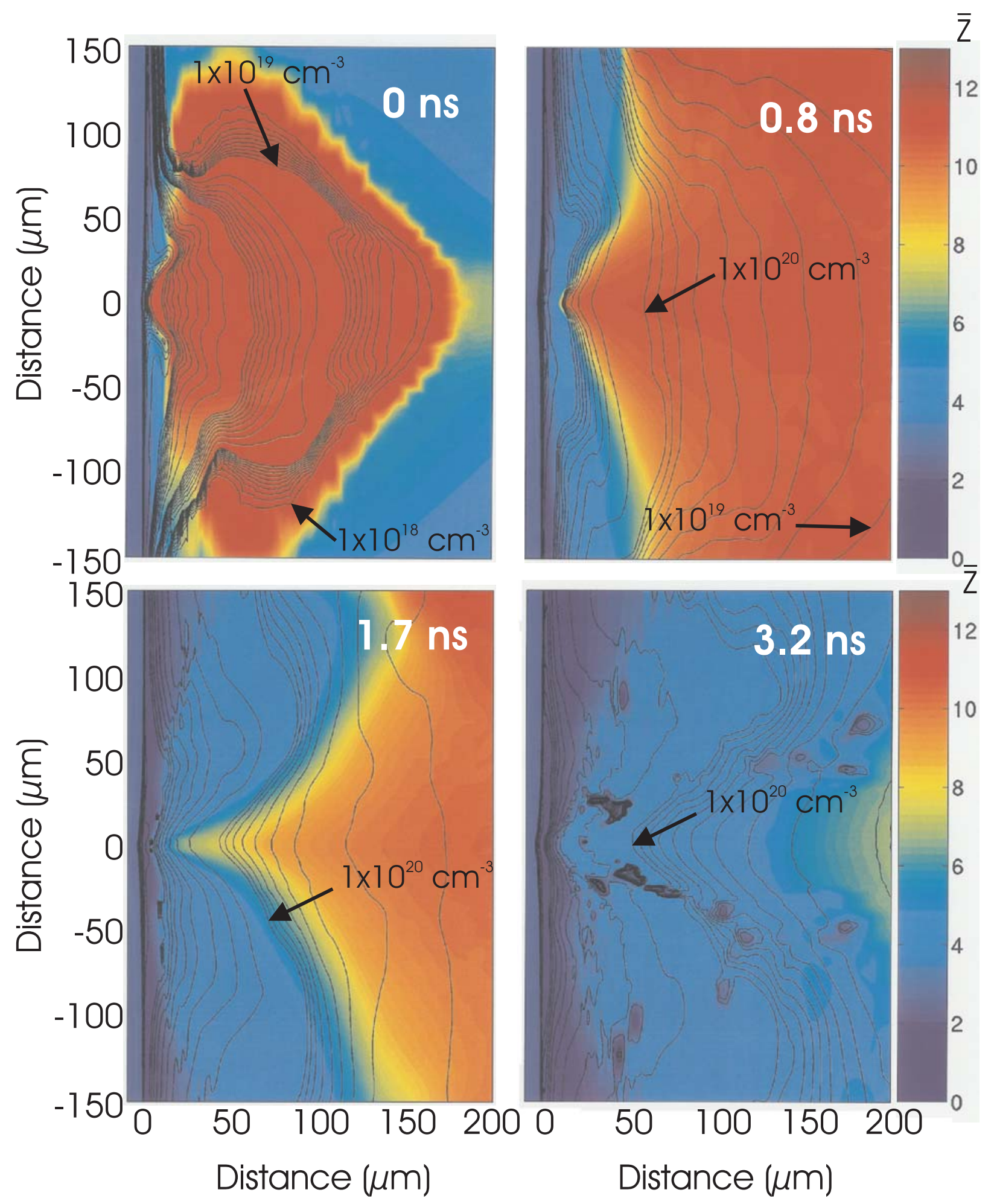

FIG. 3: Sequence of simulated electron density contours and mean ionization distribution maps computed using the LASNEX code for an Al plasma created with the irradiation conditions of Fig. 1. 


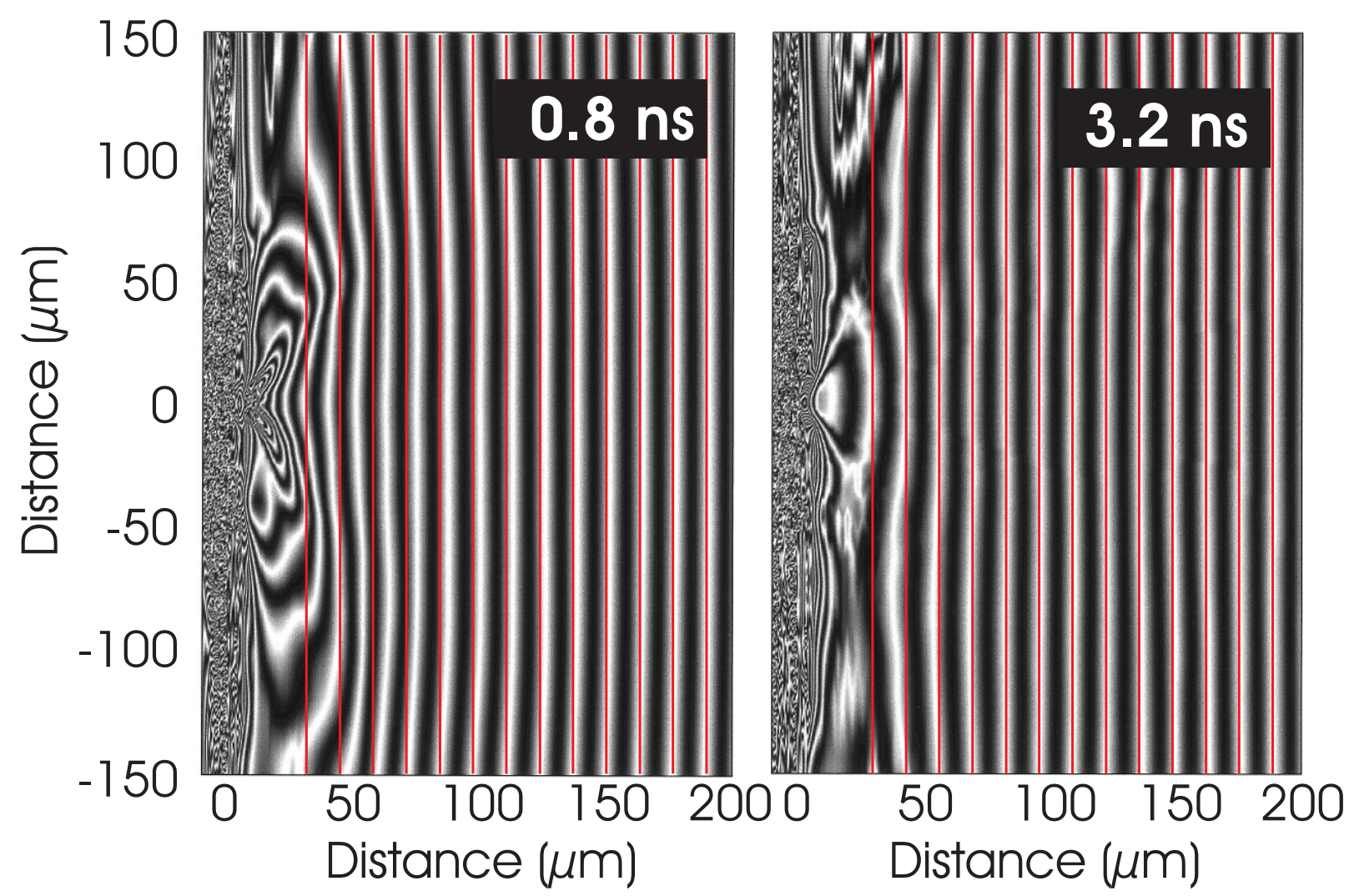

FIG. 4: Synthesized interferograms computed using the calculated electron and ion densities from Fig. 3 and the calculated scattering factors for $\mathrm{Al}$ ions. 